\section{$c 4$}

These findings suggest that immune health in adulthood can be preset by nutritional status during fetal life g

\title{
We are what mum eats!
}

New research in mice shows that exposure to vitamin A-derived retinoids in the womb determines proper development of secondary lymphoid organs (SLOs) and life-long immunity. These findings suggest that immune health in adulthood can be preset by nutritional status during fetal life.

SLO development occurs during embryogenesis and depends on a population of group 3 innate lymphoid cells known as lymphoid tissue-inducer (LTi) cells. In this study, analysis of mouse embryos at day 12.5 (E12.5) revealed a subset of



precursor cells with LTi cell potential (defined as ID2 ${ }^{+} \mathrm{ROR} \gamma \mathrm{t}^{+/-} \mathrm{CD} 4^{-}$), which were shown to mature into LTi cells (ID2 ${ }^{+} \mathrm{ROR} \gamma \mathrm{t}^{+} \mathrm{CD} 4^{+}$) when transplanted into organ cultures. Notably, supplementing cultures of E13.5 lymph node cells with the vitamin A metabolite retinoic acid (RA) increased the differentiation of precursors into LTi cells. Moreover, the embryos of pregnant mice that were fed an RA-enriched diet starting on E10.5 had an increased proportion of mature LTi cells at the expense of LTi precursor cells. This effect of RA exposure on LTi cell development was lost if the pregnant mice were given the RA signalling inhibitor BMS 493. Treatment with the RA signalling inhibitor led to the development of embryos with smaller lymph nodes and no Peyer's patches.

To confirm a direct effect of RA on the LTi precursor cells, the authors generated mice with targeted expression of a dominant-negative form of the transcription factor RA receptor- $\alpha$ (Rara) in LTi cells. The abrogation of RA-induced signalling in vivo was associated with reduced production of pro-inflammatory cytokines and chemokines, impaired differentiation of LTi cells and the defective development of fetal lymph nodes and Peyer's patches. In addition, Rara-mutant LTi cells had lower expression of retinoic acid receptorrelated orphan receptor- $\gamma \mathrm{t}$ (ROR $\gamma \mathrm{t}$; which is encoded by Rorc) than wild-type LTi cells. Restoring Rorc expression in these cells by retroviral transduction rescued their ability to differentiate into LTi cells and to express pro-inflammatory genes. Finally, the identification of functional RAR binding sites within the Rorc locus confirmed that RA acts directly on LTi precursor cells to promote the expression of genes that are involved in LTi cell differentiation.

To determine whether reduced RA-induced signalling in early life has consequences for long-term immunity, the authors generated chimeric mice by transferring wild-type bone marrow into lethally irradiated 2-week-old mice that had RARadominant-negative LTi cells and then infected them with murine herpesvirus 4 . In control chimeric mice, the virus was efficiently cleared by day 10 but in mice that had compromised RA signalling in early life only, $\mathrm{CD}^{+} \mathrm{T}$ cell expansion was reduced and the virus was still detectable 14 days after infection.

So, the efficiency of immune responses to infection may be predetermined in early life through maternal dietary signals. Whether RA exposure in utero affects responses to other immune insults or the outcome of chronic inflammatory diseases remains to be determined.

Lucy Bird

ORIGINAL RESEARCH PAPER van de Pavert, S. A. et al. Maternal retinoids control type 3 innate lymphoid cells and set the offspring immunity. Nature 508, 123-127 (2014) 